\title{
Einleitung
}

Neben einer berechtigten Faszination für die "neuen Medien" und die hieraus resultierenden Kommunikations-, Lern- und Erlebniswelten wird immer wieder die gesellschaftliche Frage aufgeworfen, ob sich bestimmte Angebote und Nutzungsgewohnheiten auch nachteilig auf die Rezipienten und ihre Entwicklung auswirken können. Hier lassen sich im Wesentlichen zwei kritische Betrachtungsweisen voneinander abgrenzen: Die quantitative Perspektive, in welcher die mit Bildschirmmedien verbrachte Zeit als mögliche problematische Einflussgröße gesehen wird und die qualitative Perspektive, bei der gerade hinsichtlich bestimmter Bildschirmmedieninhalte oder Darstellungsformate ein negatives Wirkpotential auf den Rezipienten befürchtet wird.

Eine besonders prominente Debatte innerhalb der qualitativen Sichtweise betrifft das Wirkpotential von Gewaltdarstellungen in bildschirmbasierten Unterhaltungsmedien. Gestützt auf lernpsychologische Theorien sowie eine Vielzahl empirischer Daten aus Experimenten, Querschnitts- und Längsschnittuntersuchungen wird inzwischen in den empirischen Medienwissenschaften kaum mehr angezweifelt, dass die Rezeption von Mediengewalt sowohl kurz- als auch langfristig aggressionsnahe Konstrukte in negativer Weise beeinflussen kann. Ein weiterer inzwischen ebenfalls prominenter Forschungszweig betrifft die Frage nach dem Einfluss von Unterhaltungsmedien auf die kognitive und schulische Leistungsentwicklung. Hier wurden in der Vergangenheit sowohl stimulierende als auch leistungsmindernde Annahmen vertreten und in vielfältigen Studien, gerade zum Fernsehen, systematisch untersucht. Die empirische Datenlage weist dabei inzwischen mehrheitlich darauf hin, dass ein negativer Zusammenhang zwischen Unterhaltungsmediennutzung und Schulleistungen besteht. Dies zeigt sich für erhöhte Nutzungszeiten, insbesondere jedoch für die Nutzung unterhaltungsorientierter und gewalthaltiger Angebote.

Die Gründe für diesen Zusammenhang sind jedoch nach wie vor weitestgehend ungeklärt. So wird etwa die Annahme vertreten, dass die mit Unterhaltungsmedien verbrachte Zeit für den negativen Zusammenhang ausschlaggebend ist, indem nicht-mediale Aktivitäten wie das Hausaufgabenmachen oder andere lernförderliche Alltagserfahrungen zugunsten der Mediennutzung reduziert werden. Eine andere Vermutung besteht darin, dass die Nutzung gewalthaltiger Medien langfristig zu einer Ausprägung aggressiver und einer Reduzierung prosozialer Verhaltensweisen beiträgt. Dadurch könnte, vermittelt über einen zunehmenden Rückzug aus 
der "Leistungsgesellschaft", die Partizipation an schulischen Leistungsanforderungen beeinträchtigt werden.

Für den negativen Zusammenhang zwischen Bildschirmmediennutzung und Schulleistung könnten jedoch auch Wirkmechanismen verantwortlich sein, welche schulbezogene Informationsverarbeitungsprozesse ganz unmittelbar betreffen. Mediale Unterhaltungsformate und hierbei insbesondere actionorientierte Angebote sind in der Regel durch schnelle Bildwechsel und Handlungsfolgen sowie spezifische Darstellungsformen gekennzeichnet, die Aufmerksamkeitsprozesse in besonderer Weise binden und rezipientenseitig bestimmte kognitive Verarbeitungsstile nahelegen und einfordern. Hinzu kommen bei gewalthaltigen Unterhaltungsangeboten Visualisierungen von physischen Verletzungs- und Todesfolgen sowie ein für actionreiche Computerspiele ${ }^{1}$ charakteristischer Handlungsdruck, die eine emotionale Verarbeitung dieser Erlebnisse und Handlungsanforderungen und damit eine Vielzahl von Adaptionsvorgängen beim Rezipienten erwarten lassen. Übereinstimmend mit diesen Annahmen zeigen Untersuchungen, dass die Rezeption von gewalthaltigen Medien in besonderer Weise physiologische Erregungssteigerung, die Aktivierung bestimmter Stresshormone und intensive emotionale Erlebniszustände bedingen kann. Auf diesen Erkenntnissen, auf die sich auch bekannte und inzwischen gut erforschte Annahmen zur kurzfristigen Beeinflussung aggressionsnaher Konstrukte stützen, basieren auch eine Vielzahl kognitiver Interferenzhypothesen die postulieren, dass diese Prozesse auch einen Einfluss auf kognitive Verarbeitungsprozesse und Verarbeitungsstile nehmen könnten, die außerhalb der eigentlichen Medienrezeption stehen und für schulische Lernprozesse relevant sind. Die Forschungslage hierzu ist jedoch so divergent und die Hypothesen sind zum Teil so vage formuliert, dass eine Einschätzung der Relevanz kognitiver Interferenzhypothesen für die Erklärung des negativen Zusammenhangs von Mediennutzung und Schulleistungen bisher kaum möglich ist.

Mit dieser Arbeit wird das Ziel verfolgt in Anlehnung an zwei prominente kognitive Interferenzhypothesen, die Konzentrations-Defizit-Hypothese und die Gedächtnis-Defizit-Hypothese, einen Beitrag zu der Frage zu leisten, inwieweit die Nutzung gewalthaltiger Bildschirmmedien Informationsverarbeitungsprozesse in einer Weise beeinträchtigen kann, dass hieraus Erklärungen für den Zusammenhang von Mediennutzung und Schulleistung abgeleitet werden können. Damit lässt sich diese Arbeit im Wesentlichen zwei großen Bereichen der Psychologie zuord-

1 Der Begriff "Computerspiel" wird in dieser Arbeit als Oberbegriff aller Varianten elektronischer Bildschirmspiele verstanden, die an einem Computer oder einer Spielkonsole genutzt werden können. Computerspiele lassen sich durch folgenden allgemeine Merkmale beschreiben: Der Nutzer interagiert mit dem Spiel (in der Regel eine entsprechend programmierte Software) auf einer Spielplattform (PC, Spielkonsole) über ein Interface (Schnittstelle), welches sowohl Eingabegeräte (z. B. Gamepad oder Tastatur) als auch Ausgabegeräte (Bildschirm, Lautsprecher) umfasst. 
nen: Der erste Bereich ist die medienpsychologische Wirkungsforschung, in der die Folgen von Mediennutzung auf das Erleben und Verhalten von Menschen untersucht, beschrieben und systematisiert werden. Der zweite Bereich ist die kognitive Psychologie als Teilbereich der allgemeinen Psychologie, deren Gegenstand die menschliche Informationsverarbeitung ist und die sich dabei auch einer Beschreibung und Systematisierung von Lern- und Gedächtnisprozessen widmet.

Die Arbeit ist wie folgt aufgebaut: In Kapitel 1.1 wird im Sinne eines allgemeinen Problemrahmens die gesellschaftliche Bedeutung interaktiver und nichtinteraktiver Medien beschrieben, deren Wirkpotential Untersuchungsgegenstand der vorliegenden Arbeit ist. Hierbei wird zunächst die Verbreitung der relevanten Bildschirmmediengeräte und ihre Nutzung in verschiedenen Altersgruppen beschrieben. Dieses Kapitel wird abgeschlossen durch eine Analyse der Häufigkeit von Gewalt und aggressionsbezogenen Handlungsmustern in Computerspielen und im Fernsehprogramm.

In Kapitel 1.2 wird zunächst anhand allgemeiner Schulleistungsmodelle die Bedeutung der Medienumwelt als Bedingungsfaktor schulischer Leistungsentwicklung diskutiert und eingeordnet. Anschließend wird beschrieben, welche Erkenntnisse zu der Frage vorliegen, wie sich Mediennutzung auf die schulische Leistungsentwicklung auswirkt und inwieweit hier Unterhaltung- und Gewaltformaten eine besondere Bedeutung zukommt. Unter Rückgriff auf diese Erkenntnislage wird die Frage diskutiert, wie der negative Zusammenhang von Bildschirmmediennutzung und Schulleistung erklärt werden könnte und hierbei ein Überblick über die wichtigsten Minderungshypothesen zum Zusammenhang von Bildschirmmediennutzung und Schulleistung gegeben. Im letzten Teil dieses Kapitels wird das in dieser Arbeit verfolgte Erkenntnisinteresse in die bestehende Datenlage eingeordnet, indem ein hypothetisches Arbeitsmodell zum Zusammenhang von Mediennutzung und Schulleistung eingeführt wird.

In Kapitel 1.3 wird die aktuelle Erkenntnislage zum Einfluss von gewalthaltigen Computerspielen auf kognitive und emotionale Wirkindikatoren beschrieben. Dabei wird zunächst auf die kognitive Beanspruchung durch Computerspiele und auf besonders charakteristische Unterschiede dieses interaktiven Mediums zum Filmmedium eingegangen. Anschließend wird unter Bezugnahme auf den Forschungsstand beschrieben, inwieweit kognitive Leistungen und Fertigkeiten durch Computerspielnutzung unmittelbar beeinflusst werden können und inwieweit Erkenntnisse zu der Frage vorliegen, ob sich hier gewalthaltige und gewaltneutrale Spiele in unterschiedlicher Weise auswirken. Im dritten Teil des Kapitels werden die emotionalen Wirkungen gewalthaltiger Computerspiele behandelt. Hier wird zunächst thematisiert inwieweit gewalthaltige Computerspiele physiologische Erregungsprozesse auslösen können und wie die Intensität und Dauer dieser Erregungsvorgänge einzuordnen sind. Um die hieraus gewonnen Erkenntnisse weiter 
zu präzisieren wird anschließend ein Überblick über kurzfristige spezifische emotionale Wirkungen gegeben, die in Hinblick auf gewalthaltige Medien diskutiert und untersucht wurden. Den Abschluss des Kapitels bildet eine zusammenfassende Darstellung der Erkenntnislage zu den kognitiven und emotionalen Auswirkungen der Computerspielnutzung.

In Kapitel 1.4 wird die für diese Arbeit notwendige Brücke von der Medienwirkungsforschung zur Kognitionspsychologie geschlagen. Dafür wird zunächst ein Kurzüberblick über die zentralen Gedächtnisfunktionen gegeben und hierbei insbesondere auf die Bedeutung des Arbeitsgedächtnisses und die Konsolidierung für den Wissenserwerb eingegangen. In Fortführung der Erkenntnislage zum allgemeinen Erregungspotential von gewalthaltigen Computerspielen erfolgt eine kurze biopsychologische Beschreibung von Arousal und Stress sowie ein Überblick über die Erkenntnislage zur Beeinflussbarkeit der für diese Arbeit relevanten Gedächtnisfunktionen durch Stress und emotionale Erlebnisse. Daraufhin wird diskutiert welche objektiven Merkmale auf Seiten des Mediums und subjektiven Merkmale auf Seiten des Rezipienten das emotionale Wirkpotential von gewalthaltigen Computerspielen moderieren könnten. Dieser Teil knüpft dabei an allgemeine Erkenntnisse der Stressforschung sowie an die in Kapitel 1.3 dargestellten Daten zum Erregungspotential von gewalthaltigen Computerspielen an. Den Abschluss bildet eine Zusammenfassung des Kapitels.

In Kapitel 1.5 werden aus den bisher bestehenden und empirisch bestätigten theoretischen Konzepten Fragestellungen und Wirkhypothesen der vorliegenden experimentellen Untersuchung abgeleitet. Die Hypothesen beziehen sich dabei unter Rückgriff auf den Forschungsstand sowohl auf das emotionale als auch das kognitive Wirkpotential gewalthaltiger Medien.

Es folgt der empirische Teil der Arbeit. In Kapitel 2.1 wird das gewählte experimentelle Versuchsdesign beschrieben. Die maßgeblichen Bestandteile dieser Darstellung betreffen die Stichprobenakquise, die Operationalisierung der unabhängigen und abhängigen Variablen, den Versuchsaufbau und den Ablauf der Untersuchungen. Bereits an dieser Stelle kann auf einige Besonderheiten des gewählten Versuchsdesigns hingewiesen werden. Hier ist zunächst die Stichprobe zu nennen, in die Männer und Frauen, hochgebildete und niedriggebildete Personen sowie Personen mit unterschiedlichen Vorerfahrungen mit Computerspielen zu gleichen Teilen einbezogen wurden. Die inhaltlichen und methodischen Hintergründe der Stichprobenakquise sind Kapitel 2.1.1 zu entnehmen. Eine weitere Besonderheit bilden die in Kapitel 2.1.3 beschriebenen Treatmentbedingungen. Hierbei wurden sowohl Computerspiele (gewaltneutral vs. gewalthaltig) als auch Filme (gewaltneutral vs. gewalthaltig) eingesetzt. Damit erlaubt die Untersuchung nicht nur Aussagen zur Wirkung des Faktors Mediengewalt, sondern auch zu der Frage, ob das bei Computerspielen hinzukommende Element der aktiven Interaktion von 
Nutzer und Medium von Bedeutung für das emotionale und kognitive Wirkpotential gewalthaltiger Medien ist. Für jede Treatmentbedingung wurden drei Rezeptionsblöcke (3 x 20 Minuten) mit unterschiedlichen Angeboten berücksichtigt. Hierfür wurden vorab klare Auswahlkriterien für den gewünschten Interaktionsund Gewaltgrad des eingesetzten Stimulusmaterials erarbeitet, die in Kapitel 2.1.2 ausführlich beschrieben werden.

Im Anschluss an die Beschreibung des Untersuchungsdesigns folgt in Kapitel 2.2 der Ergebnisteil der Arbeit. In Kapitel 2.2.2 wird überprüft, inwieweit vorab identifizierte und im Rahmen des Versuchsdesigns nicht vollständig kontrollierbare Störvariablen in den Treatmentgruppen in gleicher Weise wirksam wurden. In einem sich in Kapitel 2.2.3 anschließenden Manipulationscheck wird überprüft, inwieweit die Gewaltbedingungen entsprechend der Intention auch von den Versuchspersonen als gewalthaltig empfunden wurden und ob die Medien hierbei unabhängig vom Gewaltgehalt wie gewünscht einen ähnlichen Unterhaltungs- und Schwierigkeitsgrad aufweisen. In Kapitel 2.2.4 erfolgt die Überprüfung der emotionalen und kognitiven Wirkhypothesen dieser Arbeit und in Kapitel 2.2.5 werden ergänzende quasiexperimentelle explorative Auswertungen dargestellt. Die Diskussion der Ergebnisse und ihre Einbettung in den Forschungsstand in Kapitel 2.3 bildet den Abschluss der Arbeit. 


\section{Theoretischer Teil}

\subsection{Daten zur Nutzung interaktiver und nicht-interaktiver Bildschirmmedien}

Medienwirkungsforschung ist unmittelbar verknüpft mit der Frage nach der "Wahrscheinlichkeit, auf ein entsprechendes Wirkpotential zu treffen" (Groebel \& Gleich, 1993, S. 136). Eine Darstellung von Daten zur Nutzungshäufigkeit von Bildschirmmedien sowie zur Bedeutung gewalthaltiger Angebote soll deshalb den Ausgangspunkt dieser Arbeit bilden. Hiermit wird veranschaulicht, an welchen gesellschaftlichen Problemkontext diese Arbeit anknüpft. Der Überblick konzentriert sich dabei auf die beiden in dieser Untersuchung behandelten und hinsichtlich der Gewaltmediendebatte prominentesten Bildschirmmedien: Das Computerspiel als interaktives und das Fernsehen als nicht-interaktives Medium.

\subsubsection{Geräteausstattung und Nutzungszeiten}

Mit dem Einzug des Fernsehers in die privaten Haushalte in der zweiten Hälfte des 20. Jahrhunderts und mit der in jüngerer Zeit zunehmenden privaten Verbreitung von Computer und Internet sind Bildschirmmedien zu einem wichtigen Bestandteil des alltäglichen Freizeiterlebens geworden (vgl. Huston \& Wright, 1998). ${ }^{2}$ Während die Nutzung linear-rezeptiver Bildschirmunterhaltung im Sinne des klassischen "Fernsehens" jedoch inzwischen nahezu stagniert und in jüngeren Alterspopulationen sogar rückläufig ist (Feierabend \& Klingler, 2008), gewinnen interaktive Unterhaltungsmedien fortwährend an Bedeutung. ${ }^{3}$ So verfügten im Jahr 1999

2 In diesem Kapitel werden zur Darstellung der kindlichen und jugendlichen Medienumwelt aufgrund ihrer deutschlandweiten Repräsentativität maßgeblich die Kinder- und Jugendstudien KIM und JIM des Medienpädagogischen Forschungsverbundes Südwest herangezogen. In beiden Studienarmen werden seit 1998 (JIM) bzw. 1999 (KIM) Mediennutzungsparameter von Kindern im Alter zwischen 6 und 13 Jahren (KIM) und Jugendlichen im Alter von 12 19 Jahren (JIM) erhoben. Eine Sonderschwerpunktauswertung bietet zudem, wenngleich nicht mehr ganz aktuell, Daten zu Vorschulkindern aus dem Erhebungsjahr 2003 (Feierabend \& Mohr, 2004). Hier wurde eine deutschlandweit repräsentative Stichprobe von 245 Eltern zu ihren Kindern im Alter von zwei bis fünf Jahren befragt.

3 Nach dem AGF/GFK-Fernsehpanel, in welchem die Zeiten technisch-verhaltensbasiert und nicht mittels Fragebogen erfasst werden (vgl. GfK-Fernsehforschung, 2005), verbrachten dreibis fünfjährige Kinder 1992 täglich 66 Minuten mit dem Fernsehen, ein Wert der seither nur geringfügig und mit leichter Schwankungsbreite auf 73 Minuten im Jahr 2007 angestiegen ist. Für die Altersgruppen der Sechs- bis Neunjährigen sowie der Zehn- bis Dreizehnjährigen ist 